\title{
How NKT cells detect microorganisms
}

Although natural killer T (NKT) cells that express a semi-invariant $\mathrm{T}$ cell receptor (TCR) a-chain (Va14Ja 18 in mice and the homologous Va24Ja18 in humans) recognize glycolipid antigens presented by CD1d, it is unclear which ligands activate these cells during a microbial infection. Now, two papers published in Nature show that both mouse $\mathrm{Va} 14^{+}$ and human $\mathrm{Va} 24^{+} \mathrm{NKT}$ cells recognize CD1d-presented glycosphingolipids from Gram-negative bacteria that lack lipopolysaccharide (LPS).

Previous studies have shown that Va14 ${ }^{+}$NKT cells are activated during microbial infection, but whether they are activated directly by TCR recognition of CD1d-presented microbial antigens or indirectly, by other immune cells responding to the pathogen, is a controversial issue that these two groups set out to investigate. Kinjo et al. showed that CD1d presentation of two distinct glycosphingolipid mixtures (GSL-1 and GSL-1') purified from Sphingomonas species and CD1d presentation of GSL- 1 'sA and GSL- 1 'sB (which are synthetic versions of individual GSL1 'components) stimulated cytokine

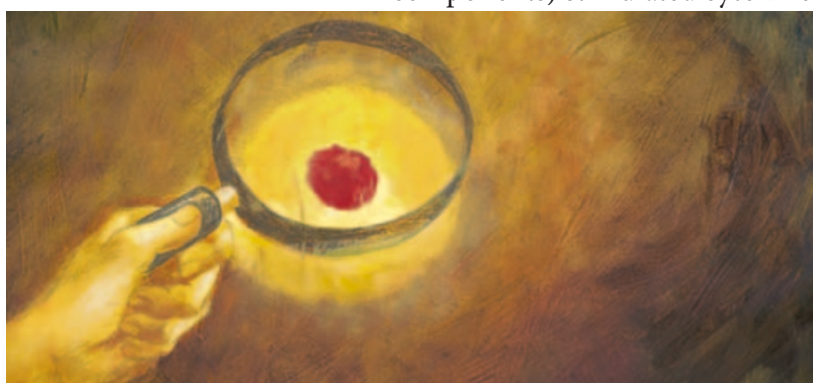

production by both human $\mathrm{Va} 24^{+}$ T-cell lines and mouse $\mathrm{Va} 14^{+} \mathrm{NKT}$ cells, but not mouse $\mathrm{T}$ cells lacking the semi-invariant Va14Ja18 TCR $\alpha$ chain. In addition, GSL-1'sA-loaded CD1d multimers bound all the human $\mathrm{Va} 24^{+} \mathrm{T}$ cells and a proportion of liver mononuclear cells from wild-type mice, but not cells from mice lacking Ja18.

Activation of $\mathrm{Va}^{+} 4^{+} \mathrm{NKT}$ cells in the liver was also observed when wild-type mice were immunized with bone-marrow-derived dendritic cells pulsed with either GSL-1'sA or live Sphingomonas yanoikuyae. This in vivo activation did not depend on Toll-like receptor (TLR) activation of the antigen presenting cells (APCs) or APC secretion of interleukin-12. Functionally, in vivo Va14 ${ }^{+}$NKT-cell activation was associated with bacterial clearance.

In a similar study, Mattner et al. showed that heat-killed Salmonella enterica serovar Typhimurium ( $S$. typhimurium), Ehrlichia muris and Sphingomonas capsulata all induced interferon- $\gamma($ IFN- $\gamma)$ production by Va14 ${ }^{+}$NKT cells. Surprisingly, the response to $S$. typhimurium (an LPSpositive Gram-negative bacterium), but not the other two bacteria (both of which are LPS-negative Gramnegative bacteria), required TLR signalling by the APCs. In addition, if $\mathrm{Va} 14^{+} \mathrm{NKT}$-cell recognition of the recently identified endogenous glycolipid ligand isoglobotrihexosylceramide (iGb3) was prevented, the response to $S$. typhimurium, but not the other two bacteria, was reduced; indicating that iGb3 activates $\mathrm{Va} 14^{+}$ NKT cells following infection with $S$. typhimurium. By contrast, CD1d presentation of synthetic versions of glycosphingolipids from Sphingomonas species stimulated IFN- $\gamma$ production by both mouse $\mathrm{Va} 14^{+}$and human $\mathrm{Va} 24^{+}$NKT cells. CD1d tetramers loaded with these compounds bound the human $\mathrm{Va} 24^{+} \mathrm{T}$ cells and a proportion of mouse $\mathrm{Va} 14^{+} \mathrm{NKT}$ cells, indicating that they are recognized directly by the NKT cells. In addition, although mice lacking $\mathrm{Va} 14^{+}$ NKT cells showed impaired bacterial clearance after infection with $S$. capsulata compared with wild-type animals, they also showed reduced lethality after high dose infection, as they lack the NKT cell population that produces high levels of cytokines in response to microbial antigens.

These studies provide clear evidence that some microbial antigens can be directly recognized by NKT cells, whereas other microorganisms are sensed indirectly through the recognition of iGb3. The authors of both papers suggest that direct recognition of microbial antigens by NKT cells may be an innate immune mechanism for detecting microorganisms that lack TLR ligands.

Karen Honey, Associate Editor, Nature Reviews Immunology

(2) References and links ORIGINAL RESEARCH PAPERS Kinjo, Y. et al. Recognition of bacterial glycosphingolipids by natural killer T cells. Nature 434, 520-525 (2005) | Mattner, J. et al. Exogenous and endogenous glycolipid antigens activate NKT cells during microbial infection. Nature 434, 525-529 (2005) 